ITEP-LAT/2002-24

KANAZAWA 02-33

\title{
The flux distribution of the three quark system in $\mathrm{SU}(3)$
}

\author{
H. Ichie ${ }^{\mathrm{a}}$, V. Bornyakov ${ }^{\mathrm{b}}$, T. Streuer ${ }^{\mathrm{c}}$ and G. Schierholz ${ }^{c}$ \\ a Institut für Physik, Humboldt Universität, Invalidenstr. 110, D-10115 Berlin, Germany \\ b Institute for Theoretical Physics, Kanazawa University, Kanazawa 920-1192, Japan \\ c NIC/DESY Zeuthen, Platanenallee 6, D-15738 Zeuthen, Germany
}

\begin{abstract}
We study the abelian color-flux distribution of the three quark system in the maximally abelian gauge on SU(3) lattices. The distribution of the color electric field suggests Y Ansatz, which might be interpreted through the dual superconductor picture as the result of the vacuum pressure in the confined phase. In order to clarify the flux structure, we investigate the color electric field in the three quark system also in the monopole part and in the photon part.
\end{abstract}

\section{Introduction}

The SU(3) lattice study on the three quark (3Q) system is important for the clarification of the baryonic structure. For more than 20 years, there is a crucial question about the long range

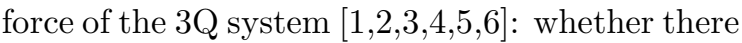
is a genuine three body force, or it can be described by the sum of the two body forces. In the former case, the flux structure is expected to be of " $Y$-shape", which has a junction at the point where the total length of strings from a quark to the junction is minimal. On the other hand, in the latter case, the flux structure is expected to be of " $\Delta$-shape", which consists of the 3 sets of two body interactions.

In order to distinguish between the two Ansätze, the $3 \mathrm{Q}$ potential was investigated using lattice simulations by several groups more than

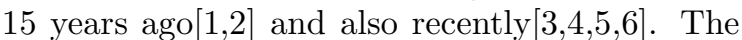
question, however, hasn't been settled yet. Some authors support the $\Delta$ Ansatz [3, th, others find evidence for the YAnsatz [6] (see also [5). This discrepancy is due to the difficulty of measuring the $3 \mathrm{Q}$ potential accurately for a large quark separation and moreover the difference between the two Ansätze in the 3Q potential is not large, at most $20 \%$. Therefore, it is useful to measure the flux directly using lattice simulations.

A couple of groups measured the flux in multi-

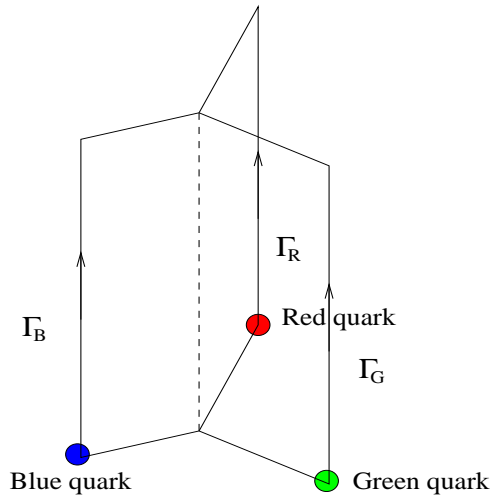

Figure 1. Three quark abelian Wilson loop.

quark systems. However, for example, the Helsinki and the UK groups [7] investigated them only in $\mathrm{SU}(2)$, where mesons and baryons have no differences, and no one has succeeded in the direct measurement of flux in the $3 \mathrm{Q}$ system in SU(3), since it needs huge statistics and large computer memory.

We shall study the flux of the three quark system in $\mathrm{SU}(3)$ after fixing the gauge into the maximally abelian (MA) gauge based on the dual superconductor scenario, because it is easy to interpret the flux structure and the validity of this scenario for the flux tube has been confirmed by Bali et al. [8]. In addition, one can get better signals on the abelian flux in the MA gauge than on the full 

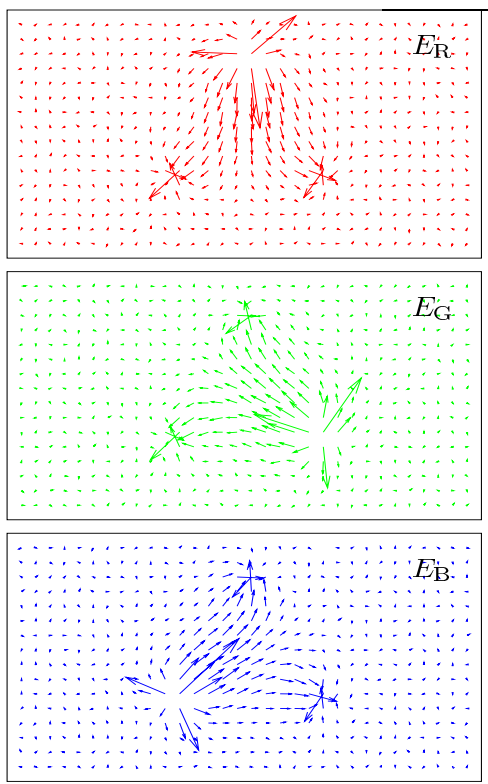

Figure 2. The three components of the color electric field. The green component $E_{\mathrm{G}}$ flows from green quark into red quark and blue quark.

flux in $\mathrm{SU}(3)$ and its computation needs only less statistics and memory than the full one, which means that there is the possibility to succeed in the measurement of the flux without waiting for a new bigger supercomputer.

\section{Operators and simulation details}

The local quantities describing the abelian flux of the $3 \mathrm{Q}$ system are obtained from the correlation between an appropriate operator and the $3 \mathrm{Q}$ abelian Wilson loop:

$O(s)=\frac{\left\langle O(s) W_{3 \mathrm{Q}}\right\rangle}{\left\langle W_{3 \mathrm{Q}}\right\rangle}-\langle O\rangle$,

where the $3 \mathrm{Q}$ abelian Wilson loop is defined by

$W_{3 \mathrm{Q}}=\frac{1}{3 !}\left|\epsilon_{a b c}\right| u_{\mathrm{R}}^{a} \cdot u_{\mathrm{G}}^{b} \cdot u_{\mathrm{B}}^{c}$

with a path product

$u_{\mathcal{C}}^{a}=\prod_{s \in \Gamma_{\mathcal{C}}} u_{\mu}^{a}(s) \quad \Gamma_{\mathcal{C}}=\Gamma_{\mathrm{R}}, \Gamma_{\mathrm{G}}, \Gamma_{\mathrm{B}}$

of abelian link variables along the path $\Gamma_{\mathcal{C}}$ in fig. 1. Differently from the nonabelian case, the color
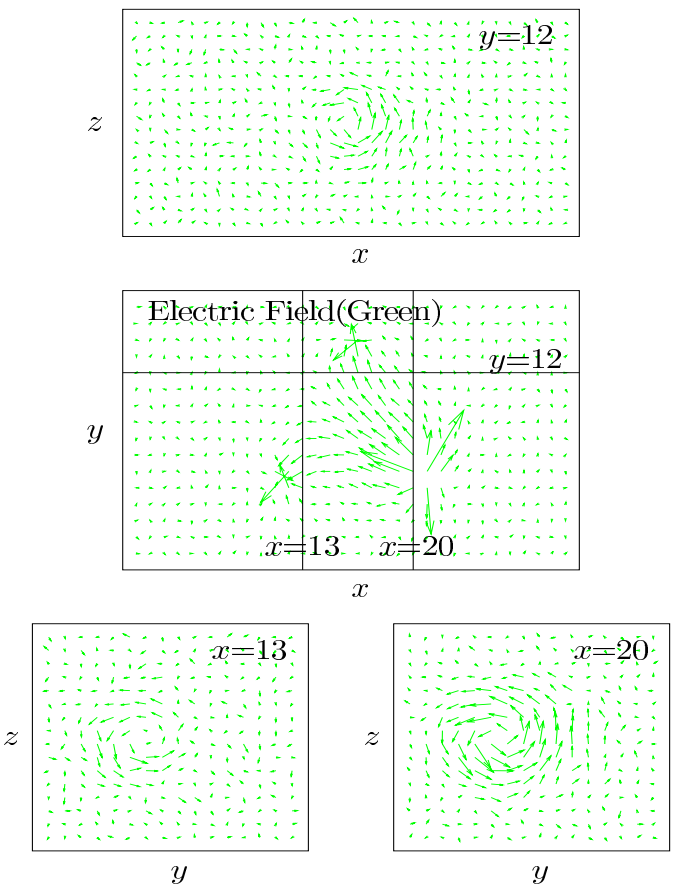

Figure 3. The magnetic super currents rotating around the color electric field (green component).

of quarks does not change during the propagation, because the off-diagonal components of the gauge field are frozen in the abelian gauge.

The simulation is performed in quenched QCD at $\beta=6.0$ on $16^{3} \cdot 32$ lattices. The link variables are fixed into the maximally abelian gauge with a simulated annealing algorithm. The 3 static charges, red, green and blue quarks, are located at $(17,14)(22,6)$ and $(12,6)$ in the $x-y$ plane, respectively. For noise reduction, we use the smearing method for spatial links of the $3 \mathrm{Q}$ abelian Wilson loop.

\section{Flux of the 3 quark system}

Figs. 2 and 3 show the color electric field and the magnetic super current in the 3Q system. For example, the green component of the electric field flows from the green quark into the blue and red quarks. For each flux, we observe a solenoidal monopole current in the plane perpendicular to 

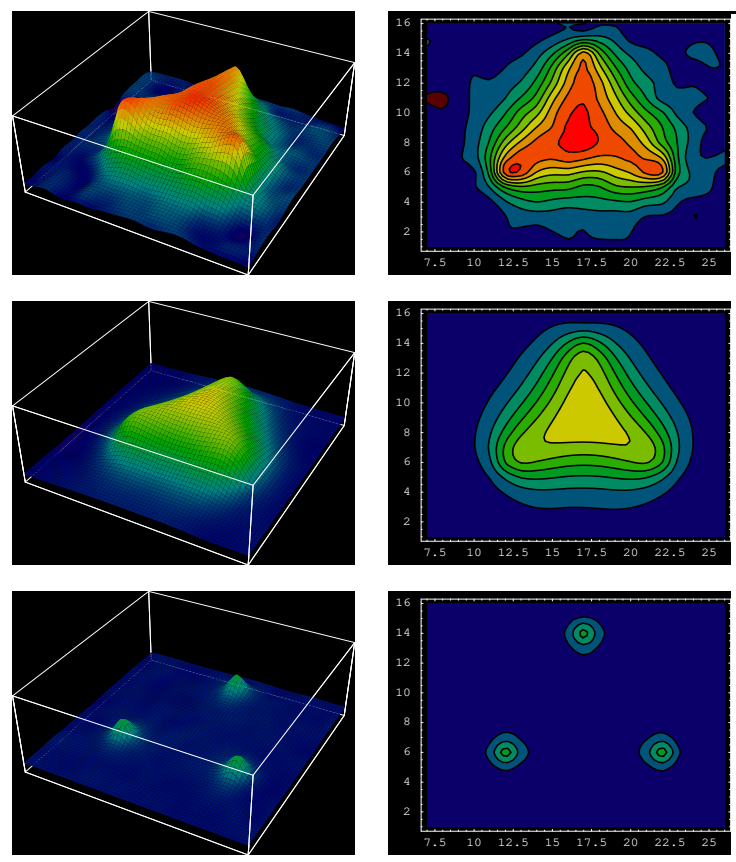

Figure 4. Action density in the abelian(top), monopole part(middle) and photon part(bottom).

the flux. Corresponding to the amount of flux of the electric field, there is a magnetic super current around each flux tube. Our results are in a qualitative agreement with dual GinzburgLandau theory [9].

In order to clarify the flux structure, we decompose the abelian gauge field into the monopole part and photon part[10]. Fig. 4 shows the action density in the abelian, monopole and photon parts, respectively. The 3 peaks originating from the sources appear in the photon part, while the plateau, which is responsible for a confinement potential, appears in the monopole part. There does not appear a dent, which is expected in the case of $\Delta$ Ansatz, in the center of the plateau. Then, the abelian action density has a clear junction in the center of the 3 quarks, which suggests YAnsatz.

As shown in fig. 5, in the monopole part, there appear two rotating induced electric fields, which cancel the electric field by original sources outside of the flux. Thus, we can expect the following scenario 10: When the three quark sources

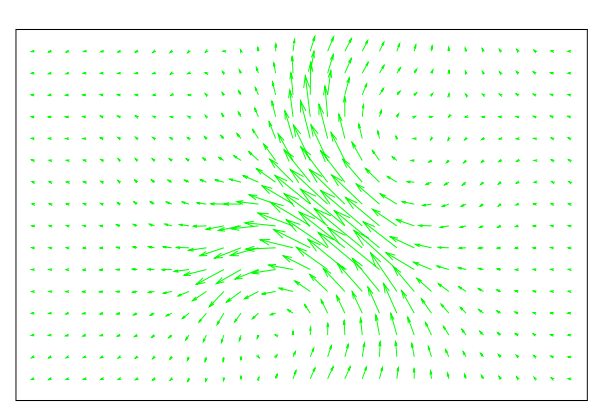

Figure 5. The induced electric field in the monopole part (green component).

are put into the vacuum, the solenoidal monopole current appears for each flux, and then they induce the two rotating electric fields such that the flux is squeezed into the " $Y$-shape".

Moreover, we investigated the flux of the $3 \mathrm{Q}$ system also in the full QCD generated with the non-perturbatively $O(a)$ improved Wilson fermion action at $\beta=5.29$ and $\kappa=0.1355[11$. For $m_{\pi} / m_{\rho} \simeq 0.7$, the flux structure is almost the same and we did not observe any big effect of dynamical quarks, like in the case of the quarkantiquark flux tube 12 .

This work is partially supported by grant INTAS-00-00111. H. I. thanks Humboldt University for hospitality. H. I. and V. B. are supported by JSPS. Computations have been done on COMPAQ AlphaServer ES40 at Humboldt University.

\section{REFERENCES}

1. R.Sommer et al.,Phys. Lett. B149 (1984)497.

2. H.B.Thacker etal.,Nucl.Phys.B(PS) (1988)234.

3. G. S. Bali, Phys. Rep. 343 (2001) 1.

4. C.Alexandrou etal.,Phys.Rev.D65(02)054503.

5. C.Alexandrou et al. these proceedings: heplat/0209062.

6. T.T.Takahashi et al., Phys. Rev. D65 (2002) 114509.

7. P. Pennanenetal.,Phys.Rev.D59(1999)014504.

8. K.Schilling et al., Nucl. Phys. B ( Proc. Suppl. ) 73 (1999) 638.

9. Y. Koma et al., Phys.Rev.D64 (2001) 014015.

10. Talk given by H. Ichie at this conference.

11. S. Booth et al. Phys. Lett. B519 (2001) 229.

12. V.Bornyakov et al. Nucl. Phys. B ( Proc. Suppl. ) 106 (2002) 634. 

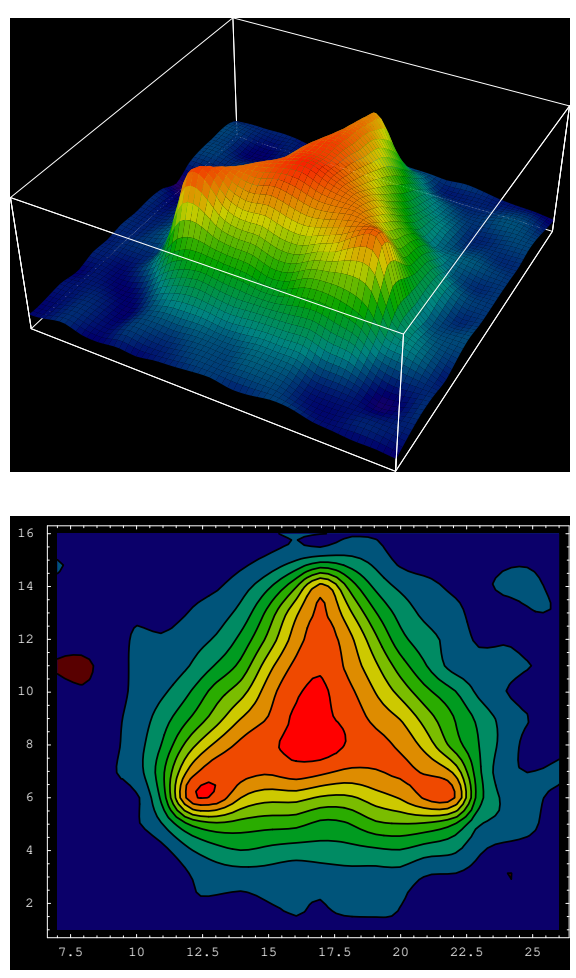
